\title{
Síndrome do Intestino Irritável: Um Exercício em Análise Funcional do Comportamento ${ }^{1}$
}

\author{
Carlos Henrique Bohm \\ Lincoln da Silva Gimenes
}

Universidade de Brasília

\begin{abstract}
RESUMO
Na Síndrome do Intestino Irritável (SII), há a alteração na frequência das evacuações intestinais associadas ao quadro de desconforto abdominal. A etiologia desse problema de saúde ainda é pouco conhecida. A literatura psicológica fornece suporte para a compreensão da SII por meio do modelo de estresse, do modelo de motilidade intestinal induzida por contingências e do modelo operante. Neste estudo foi realizado um exercício em análise funcional do comportamento com uma portadora de SII por meio de entrevistas e automonitoramento (AM) prolongado dos sintomas e das atividades diárias, para verificar a existência de relações funcionais entre esses eventos. A participante apresentava um quadro de diarreia que teve remissão durante um período de férias, em função de alteração das demandas acadêmicas. Seus sintomas pareciam adiar atividades aversivas, indicando o controle operante. Este estudo demonstrou a possibilidade de se utilizar a análise funcional como um instrumento capaz de ajudar na compreensão da SII.
\end{abstract}

Palavras-chave: análise funcional do comportamento; síndrome do intestino irritável; automonitoramento; atividades diárias.

\begin{abstract}
Irritable Bowel Syndrome: An Exercise in Functional Analysis of Behavior

Irritable Bowel Syndrome (IBS) is characterized by an alteration of the frequency of the intestinal evacuations, which are associated with abdominal discomfort. The etiology of this health problem is not very well known yet. The psychological literature offers support to IBS comprehension by stress model, adjunctive bowel motility model and operant model. This study was an exercise in functional analysis of behavior with a woman, IBS patient, through interviews and self-monitoring (SM) of daily activities and the symptoms to verify the existence of functional relations between these two events. The participant showed a diarrhea symptom which had a consistent remission during a period of vacation, as a function of changes in the academic demands. Her symptoms seemed postponing aversive activities, indicating the operant control. This study demonstrated the possibility of using functional analysis as an instrument capable of helping in SII comprehension.
\end{abstract}

Keywords: functional analysis of behavior; irritable bowel syndrome; self-monitoring; daily activities.

A Síndrome do Intestino Irritável (SII) é um transtorno gastrintestinal funcional que ocorre mundialmente, em homens e mulheres de todas as faixas etárias (Passos \& cols., 2006) e em cerca de $10 \%$ a $15 \%$ da população (Saito, Schoenfeld, \& Locke, 2002). Entre aqueles que procuram serviços médicos, há uma predominância maior em mulheres do que em homens em uma proporção de 2:1 (Chang \& Heitkemper, 2002), mas não está claro se isso reflete uma diferença real ou se as mulheres são aquelas que procuram mais ajuda médica.

A Convenção Internacional de Roma III para diagnósticos gastrofuncionais estabelece os seguintes cri- térios para a SII: Presença de dor ou desconforto abdominal que ocorra com frequência de três ou mais dias por mês, nos últimos três meses; presente há pelo menos seis meses; acompanhada de pelo menos duas características tais como, alívio com a defecação, mudança na frequência defecatória, ou mudança na forma (aparência) das fezes (Mizputen \& cols., 2006). Os pacientes da SII podem apresentar inchaço abdominal, fezes aquosas, endurecidas, eliminação excessiva de gases, dificuldades em eliminar gases e sensação de evacuação incompleta. O quadro sintomático pode ter predomínio de diarreia, de constipação ou de alternância entre os dois. Também geralmente é inclusa como 
critério diagnóstico a ausência de um substrato orgânico estrutural evidente (Passos \& cols., 2006) como inflamação, parasita ou deficiência de enzima (Whitehead \& Bosmajian, 1982) que melhor expliquem os sintomas.

Diversos prejuízos têm sido relatados nas vidas de portadores de SII, como irritabilidade, cansaço (Corney, Ruth, Robert \& Anthony, 1991), dificuldade para dormir, tensão e pouco interesse sexual (Spiegel \& cols., 2004). Na presença dos sintomas, os pacientes evitam atividades como trabalho, viagem, socialização, sexo, lazer, atividades domésticas, certos alimentos e fazer refeições com outras pessoas (Corney \& cols., 1991).

O tratamento médico para SII consiste na suplementação dietária de fibras (Blanchard, Schwarz \& Radnitz, 1987) e no uso de drogas como mebeverina, loperamida, brometo de pinavérico, amitriptilina, agentes antiespasmódicos, receptores de serotonina, ansiolíticos e antidepressivos (Quilici, Francesconi, Haddad, Passos \& Mizputen, 2006). Apesar das respostas às medicações, nenhuma medicação disponível atualmente demonstrou aliviar sistemática e globalmente os sintomas da SII (Lackner, Mesmer, Morley, Dowzer \& Hamilton, 2004). Quilici e cols. (2006) apontam que a terapia medicamentosa é ineficaz se os problemas psicossociais ficarem inalterados. Essa afirmação fortalece a ideia de que variáveis comportamentais estão envolvidas nessa síndrome.

A redução dos sintomas da SII tem sido demonstrada em estudos que utilizaram relaxamento progressivo, treino de inoculação de estresse e aprendizagem dos conceitos de assertividade (Lynch \& Zamble, 1989), bem como exercícios de relaxamento muscular progressivo, manejo de estresse, desvio de atenção de sensações físicas (relacionadas aos sintomas) e reestruturação cognitiva (Leibbrand \& Hiller, 2003). Essa redução também foi demonstrada por Neff e Blanchard (1987), que empregaram um tratamento com educação sobre o funcionamento normal do intestino, relaxamento muscular progressivo, biofeedback térmico e treinamento cognitivo de manejo de estresse. Em conjunto, essas e outras intervenções comportamentais e comportamental-cognitivas foram eficazes na modificação do estilo de vida e, por conseguinte na redução de alguns dos sintomas da SII. No entanto, esses trabalhos não apresentam descrições e análises funcionais sistemáticas das atividades diárias envolvidas nas situações que foram modificadas (geralmente denominadas de estressantes).

Existem poucos tratamentos efetivos de forma duradoura para a SII, provavelmente porque se conhece pouco sobre sua etiologia (Boyce, Gilchrist, Talley \& Rose, 2000), o que torna necessárias mais investigações sobre a mesma. De forma geral, a literatura tem indicado que as atividades da vida diária ou o estilo de vida se correlacionam com a manifestação dos sintomas.

A seguir serão descritas três das possíveis explicações para essa perturbação do funcionamento do intestino, nas quais há alusão às atividades de vida diária. A primeira é a hipótese do estresse como variável correlacionada com os sintomas da SII. A segunda é um modelo animal de motilidade intestinal como comportamento adjuntivo a (induzida por) contingências de reforçamento. A terceira é a do modelo de comportamento operante, descrevendo a manutenção dos sintomas pelas suas consequências.

Mayer, Naliboff, Chang e Coutinho (2001) definem o estresse como uma ameaça aguda à homeostase de um organismo, seja real ou percebida, que evoca respostas adaptativas para defender a estabilidade do ambiente interno e garantir a sobrevivência do organismo. Diferentes tipos de estresse exacerbam e perpetuam os sintomas gastrointestinais. Estresse a trauma no início da vida (abuso, negligência e perda dos cuidadores primários) tem um papel na vulnerabilidade dos indivíduos para desenvolver futuramente doenças gastrintestinais funcionais (como é o caso da SII). Além disso, episódios ameaçadores durante a vida adulta (transtorno do estresse pós-traumático, por exemplo) também são fatores de risco para o desenvolvimento desse problema de saúde. A exposição a situações aversivas ou pré-aversivas é a característica que concede à definição de estresse a importância da interação comportamento-ambiente.

Suls, Wan e Blanchard (1994) demonstraram, numa análise intrassujeitos, uma correlação positiva entre o estresse de um dia e os sintomas da SII que ocorreram no dia seguinte. Análises entre sujeitos indicaram que a variação no estresse não parece predizer a variação nos sintomas. Os autores discutem que, apesar de os problemas diários de vida (ou em outras palavras, as fontes de estresse) produzirem os sintomas, isso não representa o que ocorreu na maioria dos casos da sua amostra.

No estudo de Bennett, Tennant, Piesse, Badcock e Kellow (1998), a análise intrassujeitos mostrou que o estresse durante os primeiros seis meses da pesquisa foi altamente preditivo para os sintomas apresentados aos 16 meses da pesquisa. A medida de estresse utilizada foi a severidade da presença de uma ou mais dificuldades crônicas e ameaçadoras da vida diária como divórcio, dificuldades de relacionamento, doença 
séria, falhas em negócios, dificuldades em casa e cuidar de familiar com problemas emocionais ou físicos, entre outros.

Apesar dos estudos que investigam correlações entre estresse diário e sintomas da SII não evidenciarem relações causais, esses indicam a relação entre as atividades da vida diária e os sintomas da síndrome. Os resultados são conflitantes, pois há relato tanto de ausência quanto de presença de correlação entre estresse e sintomas da síndrome. A divergência nos resultados pode refletir diferentes etiologias e agentes mantenedores dessa síndrome.

Outra alternativa explicativa para a SII é o modelo experimental de comportamentos adjuntivos (ou comportamentos induzidos por contingências de reforçamento). Esses comportamentos são específicos aos esquemas de reforçamento, mas o reforço não é contingente a eles e geralmente são induzidos de forma excessiva por contingências de intervalo fixo e variável e por esquemas de liberação de estímulos (reforçadores potenciais) em tempo fixo e variável. Alguns exemplos desses comportamentos são: ingestão de água, drogas e alimento, roer materiais não comestíveis, motilidade intestinal, atividade motora, agressão, entre outros. A literatura dessa área (Gimenes, Brandão \& Benvenuti, 2005) tem sugerido aplicar os dados das pesquisas com animais para explicar transtornos comportamentais em que há ocorrência excessiva de alguns comportamentos, como o alcoolismo, tabagismo, obesidade, bulimia, síndrome do intestino irritável e transtorno obsessivo-compulsivo, entre outros.

Esse modelo tem indicado sistematicamente algumas variáveis que afetam a motilidade intestinal em animais (Gimenes, Brandão \& Benvenuti, 2005). No estudo de Gimenes, Andronis e Goldiamond (1987), a resposta de pressão à barra de ratos privados de alimento era reforçada com alimento em pó em esquema de intervalo fixo (FI) de 32 s. e esquema de reforçamento contínuo (CRF). Houve maior defecação nas sessões com FI 32 s. do que nas sessões com CRF. Em outro experimento (Gimenes, Andronis \& Goldiamond, 1988), ratos submetidos a um esquema múltiplo FI $32 \mathrm{~s}$. FI 1 s. defecaram $98 \%$ das vezes no componente FI 32 s.

Gimenes (1997) aponta que as pesquisas com animais sugerem uma análise em portadores de SII baseada na regularidade ou ciclicidade de atividades diárias como trabalho, lazer e alimentação. Uma análise de Gimenes (1997) com dois portadores de SII indicou que essa direção pode estar correta. Com base em registros de atividades cotidianas, para uma pessoa com constipação foi observada uma rotina de ativida- des caracterizada por repetições e invariabilidade ao longo do dia. Essa rotina se assemelhava a programações em razão fixa. Observou-se alteração do comportamento intestinal quando ocorreram quebras nessas rotinas. Outro relato é o caso de uma pessoa com diarreia crônica que tinha atividades diárias pontuais com tempo livre entre essas atividades. Esse padrão se assemelha a comportamentos sob o controle de um esquema de reforçamento de intervalo fixo. Foi observada a alteração do comportamento intestinal quando da alteração dessas rotinas.

Um terceiro modelo é o do comportamento operante. Neste modelo entende-se que a manifestação sintomática é mantida pelas consequências reforçadoras que a mesma produz. Latimer (1988), por exemplo, sugeriu que os sintomas e aflições de portadores de SII produzem reforçadores, tais como a evitação de responsabilidades desagradáveis e o interesse e afeição da família, amigos e provedores de cuidados com saúde.

A pesquisa de Fernandez, Perez, Amigo e Linares (1998) encontrou dados favoráveis a essa hipótese. Portadores de SII que não responderam a tratamentos medicamentosos foram divididos em quatro grupos. Um grupo experimental passou por treinamento de manejo de estresse que incluiu técnicas de relaxamento muscular progressivo, autoinstrução, resolução de problemas e enfrentamento de situações da vida diária relacionadas à sintomatologia. Outro grupo experimental foi treinado para o manejo de contingências, que consistia na extinção de comportamentos inadequados do paciente (isolamento, dependência, delegação de tarefas) na presença dos sintomas. Outras técnicas utilizadas neste grupo foram o treinamento para auto-observação, reestruturação de tempo e treinamento de habilidades sociais para obtenção de recompensas alternativas àquelas obtidas com a manifestação dos sintomas. Comparados a dois grupos controle, os dois grupos experimentais apresentaram redução significativa nos sintomas, sendo a maior redução no grupo treinado para o manejo de contingências. Esse dado sustenta a hipótese de que parte das manifestações sintomáticas da SII é mantida por reforçadores sociais como atenção.

Lackner e cols. (2004) indicam a necessidade de alterar contingências reforçadoras (atenção a episódios de dor e de outros sintomas) para o tratamento da SII. Apesar de haver esse tipo de sugestão e alguma evidência empírica, ainda são necessárias mais investigações em torno do modelo operante.

De uma forma geral, os modelos de estresse, de motilidade intestinal induzida por contingências e o operante revelam a importância de investigar a in- 
fluência das atividades diárias e do estilo de vida sobre o funcionamento intestinal. Uma das maneiras de avaliar essa relação é por meio da condução de análises funcionais dos comportamentos e dos sintomas apresentados por portadores de SII.

A análise funcional busca prever e explicar a função de um comportamento através da identificação das relações entre comportamento e ambiente. Haynes e O'Brien (1990) destacam que essas relações devem ser controláveis e Samson e McDonnell (1990) indicam que essa formulação de hipóteses pode incluir dados observáveis ou não observáveis.

A coleta de dados para a condução de análises funcionais pode ser feita por meio de observação direta, observação participante, entrevistas, questionários, testes, medidas fisiológicas e automonitoramento (AM). O último envolve a discriminação da ocorrência do comportamento e a produção do seu registro, bem como de informações adicionais (Korotitsch \& Nelson-Gray, 1999). Essa técnica é uma das alternativas mais flexíveis, baratas, e que requer poucos recursos para a coleta de dados. O AM é usado como forma de avaliação durante todo o processo terapêutico para clarificação do diagnóstico, condução de análises funcionais, delimitação dos objetivos da intervenção, planejamento de cada sessão e da intervenção como um todo, e avaliação do progresso da terapia e do seu resultado final (Korotitsch \& Nelson-Gray, 1999). Na análise do comportamento e em áreas afins, o AM tem sido utilizado para objetivos de avaliação e de terapia em uma ampla variedade de comportamentos-problema (Bohm \& Gimenes, 2008).

Nos estudos sobre SII, o AM tem sido utilizado largamente para avaliar os sintomas antes e depois de intervenções e em estudos correlacionais. Drosssman (2006) sugere o uso do AM dos sintomas da SII (seus horários, severidade e presença de fatores associados) para observar como esses sofrem influência de dietas e do estilo de vida. Resumindo, o AM é uma técnica validada para acessar tantos os sintomas da SII quanto das atividades cotidianas.

As pesquisas sobre motilidade intestinal induzida por contingências mostraram sistematicamente que a motilidade intestinal varia em função dos esquemas de liberação dos estímulos potencialmente reforçadores. Essas evidências sugerem que em portadores de SII pode ocorrer um processo semelhante, no qual os esquemas de reforçamento englobariam as atividades da vida cotidiana. Os relatos de casos clínicos, as terapias comportamentais e comportamental-cognitivas e a literatura sobre estresse e comportamento operante fortalecem a ideia de que existe relação entre atividades diárias e os sintomas da SII. Tendo em vista que a literatura carece de análises funcionais clínicas idiográficas que indiquem essa relação, esta pesquisa constou de um exercício de análise funcional do comportamento com uma portadora de SII por meio de entrevistas e AM prolongado dos sintomas e das atividades diárias.

Os objetivos desta pesquisa foram: (a) Realizar uma análise molar identificando relações funcionais entre o padrão comportamental da participante e os sintomas da SII; e (b) conduzir análises funcionais moleculares entre as atividades diárias e os sintomas, ambos registrados por meio de AM.

\section{MÉTODO}

\section{Participante}

Mediante o encaminhamento de um médico gastroentereologista do Hospital Universitário de Brasília, a participante foi convidada pelo pesquisador, via chamada telefônica, para uma entrevista inicial. Esse contato foi realizado depois da aprovação do projeto de pesquisa pelo Comitê de Ética da Faculdade de Saúde da Universidade de Brasília.

Os critérios utilizados para a inclusão da participante na pesquisa foram: a) ser diagnosticada por um médico gastroentereologista sob os critérios de Roma III; b) não estar, ou suspender tratamento medicamentoso específico para a SII durante o período de coleta de dados, com o aval do médico responsável; c) não estar em processo de psicoterapia por transtornos comportamentais ou psiquiátricos graves; d) não ser portadora de doenças orgânicas relacionadas ao trato gastrointestinal.

As sessões para coleta de dados ocorreram no Hospital Universitário de Brasília. A participante foi caracterizada com o nome fictício de Sofia. As informações de identificação da participante se referem às condições da mesma no início da pesquisa.

Dados de identificação. Sofia estava com 21 anos de idade, era solteira, trabalhava como auxiliar de escritório contábil, cursava o primeiro semestre do curso de ciências contábeis e morava em Brasília há 10 meses na casa de uma tia juntamente com o primo (filho da tia) e a companheira desta tia. A participante havia saído da casa dos pais no interior da Bahia há aproximadamente dois anos para morar com os primos em Goiânia.

Queixas da SII. A principal queixa de Sofia foi a ocorrência de cerca de três episódios diários de diarreia. 
A participante sentia frequentemente dores abdominais (que descrevia como cólicas) e urgência em evacuar. O problema havia se iniciado há dois anos, concomitantemente a sua saída da casa dos pais.

História clínica. Sofia não apresentava outros problemas de saúde e não realizou nenhum tratamento para SII.

\section{Materiais e instrumentos}

$\mathrm{Na}$ coleta de dados foram utilizados um roteiro de entrevista semiestruturada para SII (Bohm, 2009), uma adaptação do Questionário Construcional de Goldiamond (Gimenes, Andronis \& Layng, 2005) e formulários de AM para SII (Bohm, 2009).

\section{Procedimentos}

Sessões. Foram realizadas duas entrevistas iniciais semiestruturadas com duração de 90 minutos. O Questionário Construcional de Goldiamond guiou, de forma geral, a coleta dos seguintes dados: história de vida, histórico médico, tratamentos, sintomas da SII, déficits e excessos comportamentais e suas variáveis mantenedoras, repertórios comportamentais adequados, entre outros. A entrevista semiestruturada teve como objetivo coletar dados específicos e detalhados sobre: a) frequência, severidade, duração, contexto de ocorrência e há quanto tempo ocorriam os sintomas da SII e outras complicações de saúde relacionadas; b) grau de comprometimento de atividades da vida diária; c) sensações e sentimentos relacionados aos sintomas; e d) medicamentos utilizados, tempo de uso e dosagem. No primeiro encontro, a participante recebeu e assinou um Termo de Consentimento Livre e Esclarecido e recebeu informações a respeito da realização da pesquisa.

Todas as entrevistas foram gravadas em áudio. As gravações das duas primeiras entrevistas foram transcritas e as informações obtidas serviram para preparar as sessões seguintes de entrevista e para descrever os resultados da pesquisa. Em seguida foram feitas diversas sessões de entrevistas com intervalos de sete dias em média. No total, Sofia passou por 23 sessões com duração média de 70 minutos cada uma.

As sessões posteriores às sessões de entrevistas iniciais tiveram os objetivos de: (a) fazer uma leitura dos registros nos formulários de AM de cada semana; (b) esclarecer pontos confusos nos registros; (c) corrigir erros nos mesmos; (d) consequenciar positivamente registros feitos de maneira correta; (e) esclarecer aspectos gerais da vida diária que não eram registrados e (f) consequenciar positivamente comportamen- tos compatíveis com AM acurado, tais como: seguir as instruções de preenchimento dos formulários e relato de execução dos registros temporalmente próximos à ocorrência dos comportamentos. Esse reforço verbal potencial consistia em: elogios, explicação da importância do AM para a avaliação comportamental e informação de que o engajamento da participante na pesquisa seria importante para o desenvolvimento de pesquisas futuras que talvez pudessem resultar em benefícios nas vidas de outras pessoas.

Depois das entrevistas, o pesquisador fazia um registro com os aspectos mais relevantes da sessão. As informações desses relatos tiveram a mesma função das transcrições de áudio das entrevistas iniciais.

Automonitoramento. O período de coleta de dados por meio do AM foi dividido em três fases. A definição dessas fases ocorreu durante a coleta de dados a partir da análise dos mesmos. Houve inicialmente 100 dias de AM durante um primeiro período acadêmico letivo, seguidos de 34 dias de férias acadêmicas e, por fim, 23 dias em um segundo período letivo. Sofia interrompeu o procedimento de AM duas vezes, durante quatro e sete dias cada vez, sem comunicar o pesquisador com antecedência, justificando que estava muito ocupada naqueles dias.

No final da segunda entrevista, a participante recebeu treino sobre como preencher os formulários de Registro de Atividades Diárias, Registro do Funcionamento Intestinal e Registro Alimentar (recebeu instruções, em seguida fez alguns registros e o pesquisador avaliou os mesmos). A participante foi instruída para fazer registros até o final da pesquisa.

A criação do formulário para registro de atividades diárias se baseou nos formulários de registro de Goldiamond (1974). Nesse formulário foram registradas atividades diárias (refeições, atividades físicas, estudo, trabalho, lazer e outras que fossem avaliadas como relevantes), seus horários e durações, pessoas presentes, eventos que ocorriam após essas atividades e sentimentos e sensações envolvidas.

A confecção do registro do funcionamento intestinal se baseou em: a) revisões da literatura sobre AM, avaliação comportamental e SII; b) dados obtidos nas entrevistas; c) avaliação constante dos dados coletados com o formulário; e d) reformulações da estrutura do formulário, com base nessas avaliações. Nesse formulário, era feito pelo menos um registro por dia, assinalando a ocorrência de alguns sintomas da SII. Foi registrada cada evacuação, seu horário e duração, local e aspecto das fezes. Quando ocorria mais de uma evacuação no mesmo dia, um novo registro deveria ser feito. 
O registro do consumo alimentar foi inserido durante a pesquisa porque se percebeu a necessidade de avaliar a relação entre o consumo de fibras e os sintomas da SII. Nesse formulário foi registrado o tipo de refeição, os alimentos e bebidas consumidos e a quantidade de cada um. Sofia fez esse registro por um período de 14 dias, tendo sido iniciado no $77^{\circ}$ dia (dias contados a partir do início dos registros de atividades diárias e do funcionamento intestinal). Uma nutricionista avaliou a precisão das medidas dos alimentos e da descrição dos tipos de alimentos. Depois dessa avaliação o pesquisador esclareceu alguns registros imprecisos com a participante.

Entrevista devolutiva. Após o término da análise dos dados da pesquisa, foi conduzida uma sessão em que a participante recebeu a análise dos seus dados e pode expressar suas opiniões sobre a pesquisa e esclarecer possíveis dúvidas.

\section{Análise dos dados}

Categorização e análise de atividades diárias. Foram selecionadas algumas atividades que pareciam não ocorrer todos os dias, isto é, aquelas atividades que sofriam variações. Suas definições se basearam nos registros da participante e nas informações obtidas durante as entrevistas. Foram estimadas as durações das ocorrências de cada categoria de atividade. Alguns registros continham a descrição de mais de uma atividade na mesma entrada do registro (ou seja, no mesmo horário). Nesses casos o tempo total do registro foi dividido pelo numero de atividades descritas, e esse valor foi utilizado como a duração da atividade. Foram escolhidas cinco categorias de atividades e foi realizada a análise da distribuição, ao longo do dia, da duração das atividades e da ocorrência da evacuação intestinal. As categorias foram: "aula/prova na faculdade", "fazer trabalho da faculdade/estudar", "atividades fora do escritório em horário de trabalho para fazer coisas do trabalho", "atividades fora do escritório em horário de trabalho para fazer coisas pessoais" e "atividades recreativas e socializadoras". Somente a última categoria demonstrou alguma relação com a evacuação.

Análise da relação entre consumo de fibras e sintomas da SII. A partir dos registros alimentares, uma nutricionista mensurou o consumo diário de fibras totais, de fibras solúveis e de fibras insolúveis. Essa mensuração foi feita por meio de tabelas de composição de alimentos utilizadas por nutricionistas. Foram realizados cálculos de correlação entre o consumo de fibras de cada dia e os sintomas do mesmo dia e do dia seguinte.

\section{RESULTADOS}

Inicialmente será descrito o perfil/padrão comportamental predominante da participante (cuja fonte de dados foram as entrevistas), relacionando-o com os sintomas da SII. Em seguida, serão mostrados alguns dados coletados com o AM que confirmam e expandem algumas relações funcionais.

Sofia tinha um quadro de diarreia, dor abdominal e urgência em evacuar. Esses sintomas pareciam ter iniciado há dois anos, na mesma época em que saiu da casa dos seus pais. Em relação a outros problemas além da SII, a participante tinha dificuldade em expressar desacordo de opiniões alheias e negar pedidos abusivos. Sua fala era excessivamente rápida e não olhava na direção do ouvinte. Relatou estado de excessiva preocupação e ansiedade em relação ao cumprimento de tarefas acadêmicas (para as quais sua mãe exigia alto desempenho) e do trabalho. A participante relatou também sentimentos de tristeza e solidão devido à falta dos amigos e familiares que viviam distante. Quando aumentavam suas demandas acadêmicas (provas e trabalhos), tinha pensamentos obsessivos e catastróficos sobre relacionamentos amorosos, vida acadêmica e familiar. Nesses períodos ocorriam também os comportamentos de roer unha, ingestão excessiva de alimento, dificuldade para dormir e posterior sono e cansaço no dia seguinte, diarreia e mais raramente o aparecimento de manchas roxas na pele. Sofia relatou a constante necessidade de "desabafar com as pessoas" porque era "muito carente". Esse comportamento verbal consistia no relato de sua ansiedade e dos seus pensamentos ruminativos.

Sofia muitas vezes fazia os trabalhos acadêmicos intercalando-os com as atividades do trabalho. Queixava-se de falta de entendimento do conteúdo ensinado pelos professores, de falta de conhecimento para realizar as tarefas extraclasse e de dificuldade para se concentrar durante as leituras. Durante a pesquisa solicitou algumas vezes ao pesquisador a suspensão temporária do procedimento de AM devido à falta de tempo. Muitas vezes, os aumentos das demandas de atividades acadêmicas e de trabalho provocavam atrasos a alguns compromissos.

Em resumo, Sofia apresentava os seguintes comportamentos-problema: atrasos a compromissos, rotina de estudos desorganizada e dificuldade em planejar e executar a resolução de problemas do dia a dia. Esse repertório se mostrava insuficiente para atender certas demandas de trabalho e do curso, o que tornava essas atividades aversivas, isto é, o não atendimento das 
demandas resultava em insatisfação dos seus superiores no trabalho e da sua mãe (em relação às notas da faculdade). Esse padrão comportamental parecia estar correlacionado com a ocorrência dos sintomas da SII (diarreia e dor abdominal).

A Figura 1 apresenta o número de ocorrência diária de evacuações da participante Sofia em cada fase do AM. Ao longo do primeiro período letivo, o número de evacuações aumentou gradativamente, indicando que o aumento das atividades acadêmicas ao longo do semestre letivo correspondeu ao aumento gradual da frequência de evacuação. Durante o início do primeiro período letivo inicial (66 dias), o número de evacuações foi menor do que no final do mesmo (34 dias). Nas férias acadêmicas, as evacuações diminuíram, comparando com o final do primeiro período letivo. No segundo período letivo, aumentou a ocorrência de evacuação, comparando com as férias. Nota-se ainda na Figura 1 que no final do período de férias aumentou o número de evacuações, o que sugere um possível controle pela proximidade do início do segundo período letivo. Isto é, a proximidade temporal do início de um novo período acadêmico provavelmente teve função de estímulo pré-aversivo.

O mesmo padrão de resultados da Figura 1 foi observado quando se analisou a porcentagem de evacuações que foram precedidas pela sensação de urgência em evacuar e as porcentagens de ocorrência de cada aspecto das fezes (normais, amolecidas ou aquosas). Isto é, nos períodos de atividades acadêmicas a porcentagem de evacuações que foram precedidas pela sensação de urgência em evacuar foi maior, a porcentagem de fezes normais foi menor e a porcentagem de fezes aquosas foi maior. Essas relações indicam que a retirada das demandas acadêmicas atuou na normalização dos sintomas da SII.

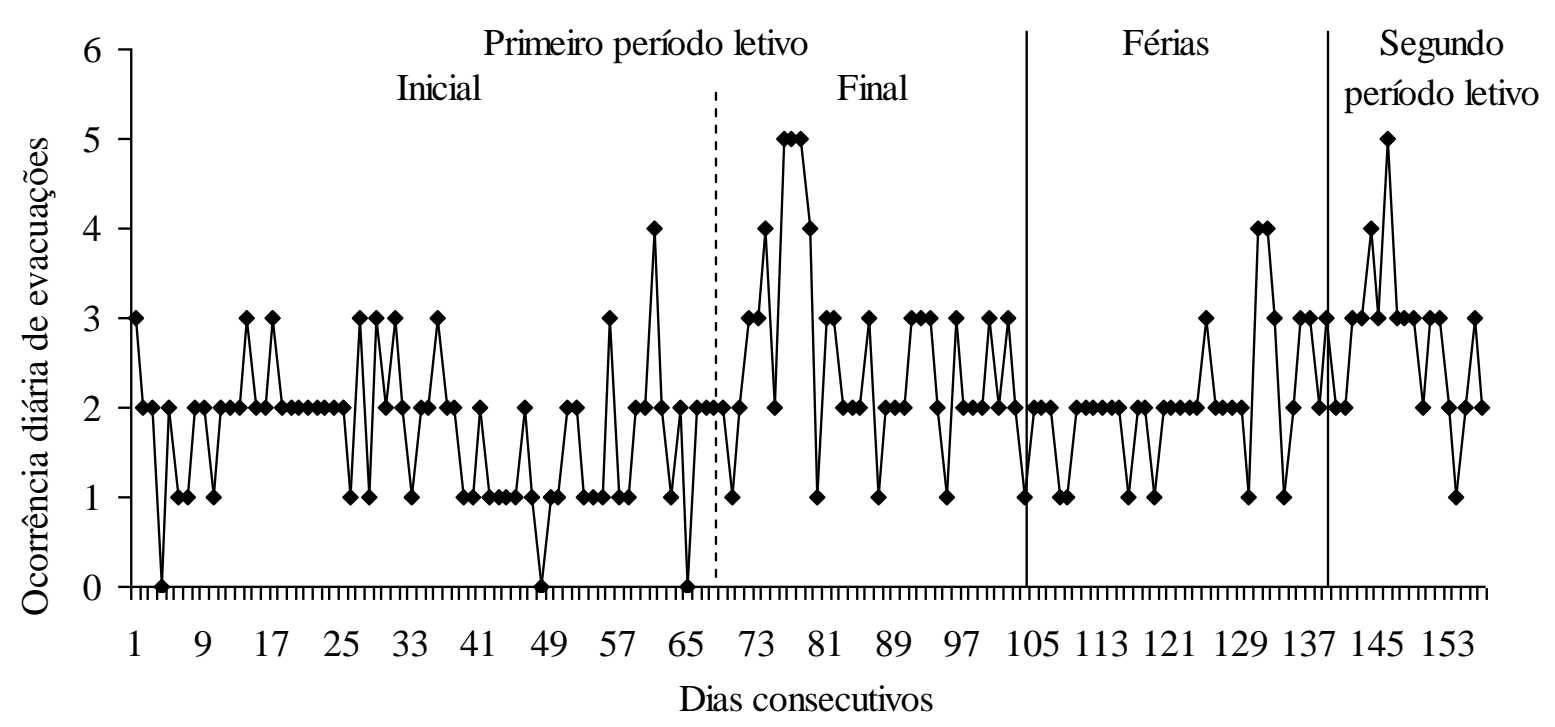

Figura 1. Número de ocorrência diária de evacuações da Participante Sofia, em cada fase do AM.

A Figura 2 mostra a distribuição ao longo do dia da média dos minutos gastos por dia com atividades recreativas sociais e da média de evacuações por dia em cada fase do AM, em blocos de 30 minutos, para a participante Sofia. O painel superior mostra que no final do primeiro período letivo (incluindo apenas os últimos 34 dias do período letivo) houve um pico de ocorrência de evacuação intestinal no início da manhã, antes da participante Sofia sair de casa para trabalhar, obrigando-a a permanecer em seu domicílio pelo tempo suficiente para se sentir segura para evitar a sensa- ção de urgência em evacuar. Essas evacuações resultavam em frequentes atrasos a compromissos do trabalho e às sessões de entrevista desta pesquisa. No final da tarde, antes dela sair do escritório onde trabalhava, ocorreram nas três fases outro pico de evacuações que parecia ter a mesma função de evitar a sensação de urgência em evacuar, e, além disso, de evitar a necessidade de usar um toalete público. O pico de evacuação do início da manhã diminuiu no período de férias acadêmicas (painel central) e voltou a aumentar durante o segundo período letivo (painel inferior). 


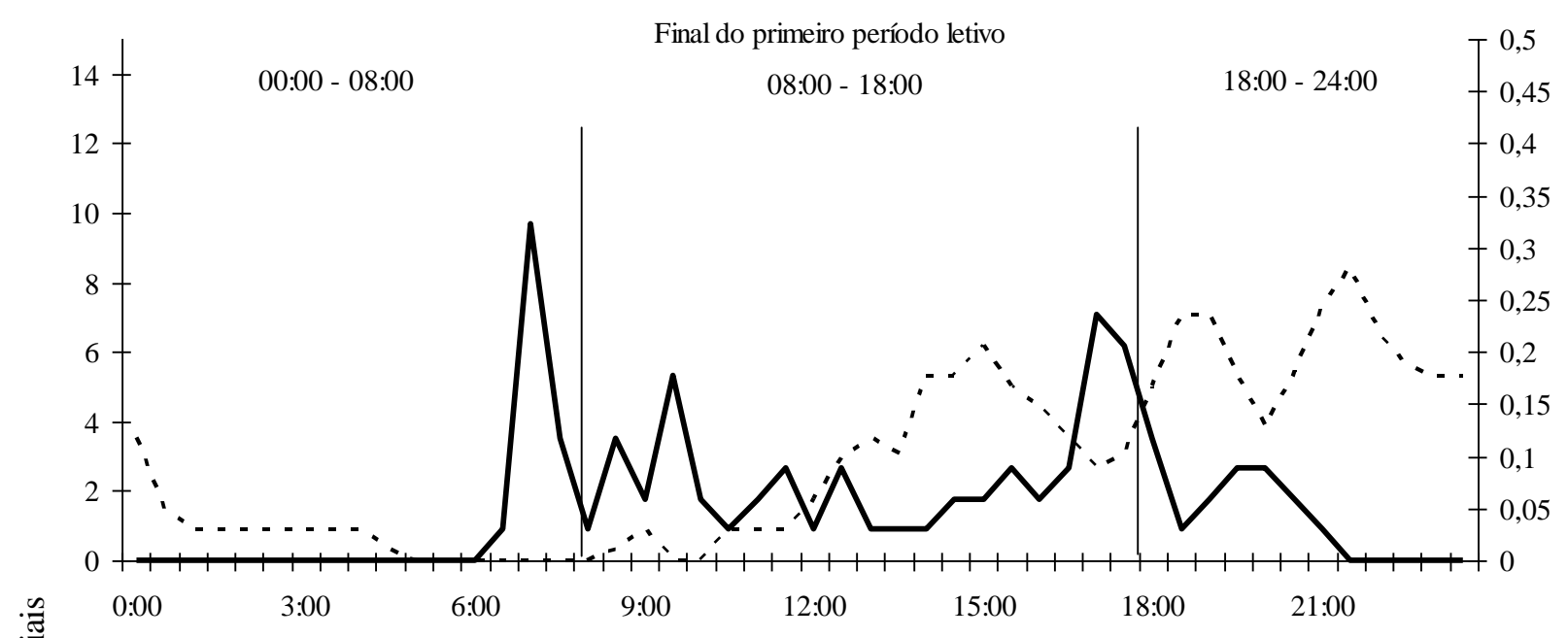

政

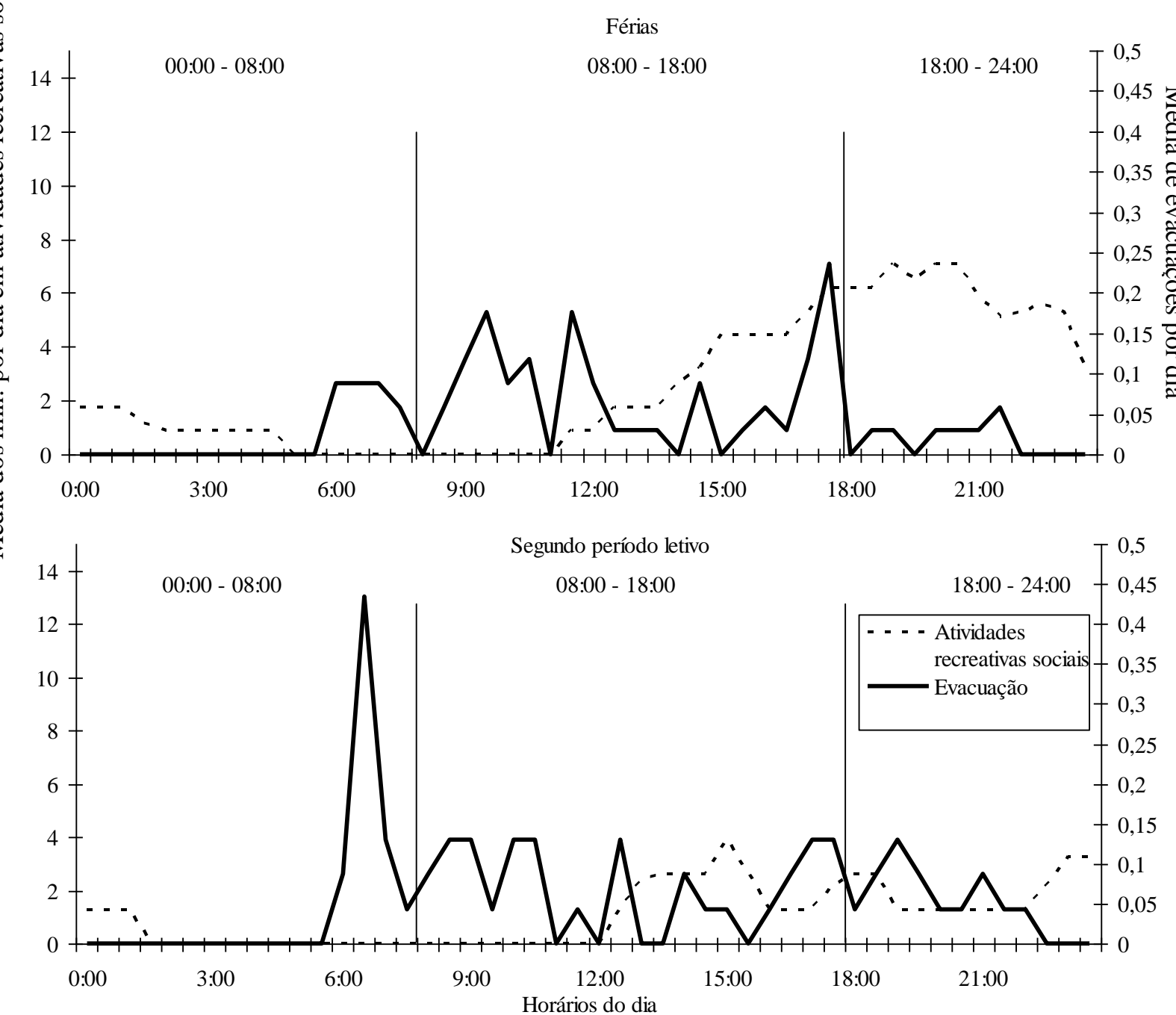

Figura 2. Distribuição ao longo do dia da média dos minutos gastos por dia em atividades recreativas sociais e da média de evacuações por dia em casa fase do AM, para a participante Sofia, em blocos de 30 minutos. O painel superior mostra os dados do final do primeiro período letivo, o painel central das férias e o painel inferior do segundo período letivo. 
Ainda na Figura 2, observa-se nas três fases uma relação inversa entre a ocorrência de evacuação e as atividades recreativas socializadoras, com exceção do ponto às 17:00 horas no período das férias. Quando ocorreram estas atividades, geralmente a evacuação foi menor do que quando as atividades não ocorreram. Os dados dessa figura tornam plausível a hipótese de haver uma relação funcional entre o excesso de evacuações em determinados horários do dia e as atividades aversivas acadêmicas e de trabalho.

Não foram observadas correlações estatisticamente significativas entre o consumo de fibras e os sintomas. Em resumo, o problema do funcionamento intestinal da participante Sofia parecia ter sido mantido por controle aversivo, no sentido de que as evacuações eram eliciadas por estímulos pré-aversivos (principalmente relacionados com atividades acadêmicas) e eram consequenciadas com o afastamento temporário da estimulação aversiva. E ainda, na fase de férias acadêmicas, a remoção da estimulação aversiva normalizou o funcionamento intestinal.

\section{DISCUSSÃO}

As relações funcionais observadas no caso da participante Sofia são favoráveis a uma explicação por meio do modelo operante. $\mathrm{O}$ seu repertório acadêmico e de trabalho (desorganização, falta de planejamento e dificuldade em resolução de problemas) parecia ser insuficiente para o cumprimento de algumas atividades. A sinalização das demandas que ela deveria cumprir (solicitações de tarefas pelos seus superiores no trabalho, prazos de entrega de trabalhos e de realização de provas na faculdade) aumentava o número de evacuações diarreicas e estas tinham como consequência a eliminação temporária do contato com essas atividades. Os relatos dos sintomas e de outras queixas (o "desabafar") tornavam as pessoas mais solidárias e compreensivas (alguns colegas faziam trabalhos para ela e os seus comportamentos inadequados eram provavelmente menos punidos) e também eliminavam temporariamente o contato com essas atividades aversivas. As pesquisas de Whitehead, Renault e Goldiamond (1975) e Fernandez e cols. (1998) dão suporte à interpretação da evacuação excessiva de Sofia como um operante.

No estudo de Whitehead, Renault e Goldiamond (1975), quando dinheiro foi liberado contingente ao aumento da secreção gástrica em um esquema de reforçamento diferencial de taxa alta, a taxa de secreção aumentou significativamente em relação à linha de base. Quando o dinheiro dependeu do decréscimo da secreção em um esquema de reforçamento diferencial de outros comportamentos, a taxa de secreção retornou aos níveis da linha de base. Esse experimento mostra sistematicamente que a secreção gástrica pode ser controlada pelos procedimentos de condicionamento operante e apóia a noção de controle consequencial observado com a participante Sofia, uma vez que tanto a produção de suco gástrico quanto a movimentação intestinal são atividades que ocorrem no aparelho digestivo e associadas ao sistema nervoso autônomo.

O experimento de Fernandez e cols. (1998) demonstrou, com o treinamento de manejo de contingência, que parte das manifestações sintomáticas da SII é mantida por atenção e privilégios. O caso da participante Sofia corrobora esses dados, evidenciando que alguns sintomas da SII ocorrem sob controle operante. A Figura 3 apresenta um diagrama com algumas possibilidades de contingências relacionadas às atividades diárias e aos sintomas da participante Sofia. Esse diagrama é baseado no paradigma da expansão da contingência para compreensão do comportamento, conforme Gimenes (2003).

Outro dado importante observado nesta pesquisa se refere à composição da dieta. Como não foi observada nenhuma correlação estatisticamente significativa entre o consumo de fibras e os sintomas da SII, é mais plausível a ideia de haver relação funcional entre o funcionamento intestinal de Sofia e variáveis ambientais, tais como o tipo e o padrão de atividades realizadas.

A literatura sobre controle aversivo (Sidman, 2003/1989) e sobre estresse (Bennett \& cols., 1998; Mayer \& cols., 2001) tratam esses dois processos como fenômenos distintos. O caso de Sofia indicou que os sintomas da SII estiveram sob controle aversivo, e também se pode afirmar que a participante vivia sob situações de estresse. No entanto, essas duas etiologias da SII (estresse e controle aversivo) parecem se referir aos mesmos processos psicológicos básicos. Dessa forma, o confronto dos dados dessa pesquisa com a literatura pode levar futuramente a propostas de articulações entre a noção de estresse e de controle aversivo, verificando o que há de semelhante nos dois modelos para melhor compreender a origem e a manutenção da SII.

Deve-se notar ainda que a medida de frequência de episódios de evacuação é uma medida indireta da motilidade intestinal. Como a participante sofria de um quadro de diarreia, poderia ter movimentações 
intestinais sem a ocorrência de evacuação devido ao esvaziamento intestinal e ausência de massa fecal. $\mathrm{O}$ caráter indireto dessa medida não permite medir com precisão absoluta a movimentação intestinal. Nesse caso, os movimentos intestinais podem, entretanto, ser detectados através do relato verbal de dores abdominais do tipo cólicas. Apesar dessas limitações, as análises funcionais permitem, em nível macro, o estabelecimento de relações funcionais entre o comportamento intestinal e variáveis ambientais.
Semestre letivo

Potenciação

Repertório acadêmico insuficiente

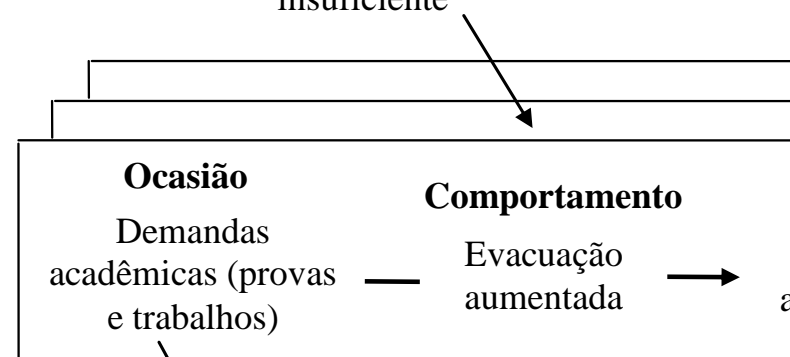

\section{História}

Exigência familiar por alto desempenho acadêmico

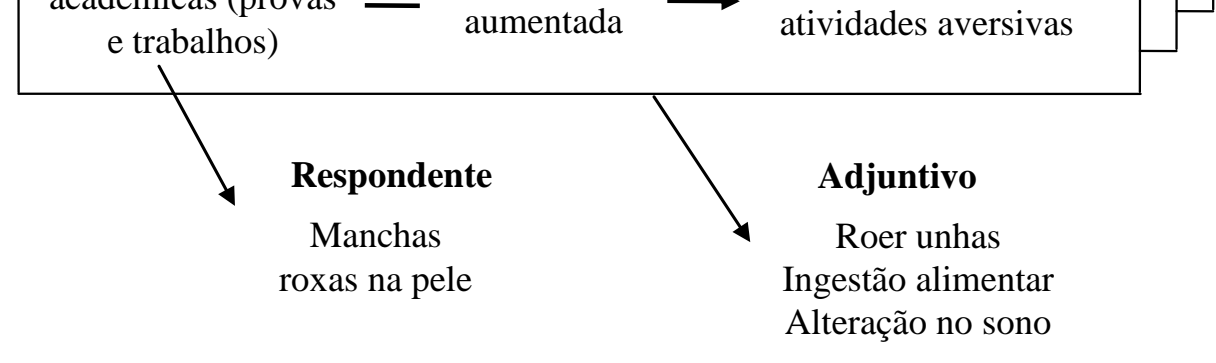

Férias acadêmicas

Potenciação

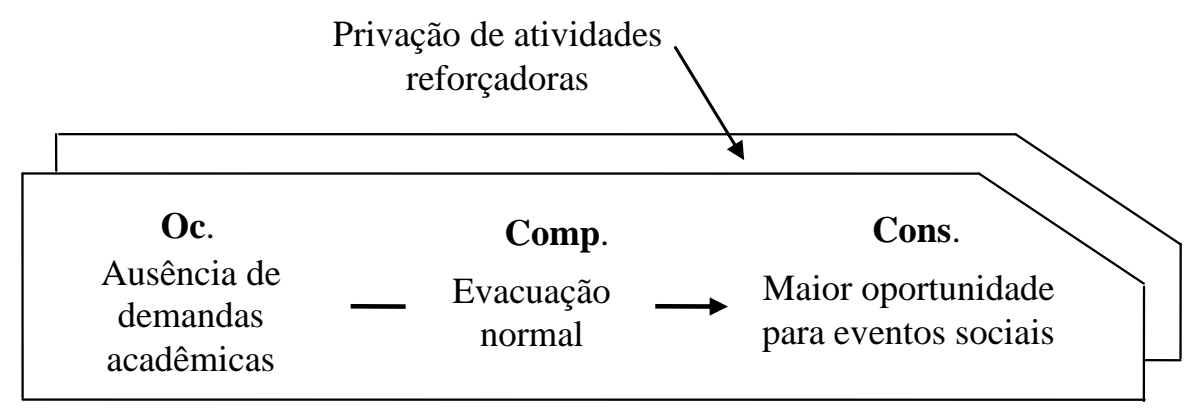

Figura 3. Possibilidades de contingências relacionadas às atividades diárias e aos sintomas da participante Sofia.

Os procedimentos empregados nesse estudo permitiram a condução preliminar de análises funcionais, tendo em vista o pouco conhecimento que se tem sobre as relações funcionais entre os sintomas da SII e as atividades diárias. Pesquisas futuras poderão aperfeiçoar os procedimentos de coleta de dados para esse grupo nosológico.

Uma das possibilidades de coleta de dados é o uso de instrumentos padronizados. Tendo em vista que é difícil medir relações funcionais e obter confiabilidade e validade dos julgamentos do clínico em uma análise funcional, os instrumentos de avaliação padronizada (escalas, inventários e protocolos) ajudam o profissional a detectar as relações funcionais (Virués-Ortega \& Haynes, 2005). Apesar de existirem instrumentos para análises funcionais de outros tipos de transtornos, como comportamento de autolesão, agressão e estereotipias (Paclawskyj, Matson, Rush, Smalls \& Vollmer, 2000), não se conhece nenhum instrumento para a condução de análises funcionais específicas para portadores de SII.

Possíveis aplicações desta e de futuras pesquisas poderão contribuir para o esclarecimento do diagnóstico da SII, pois esta é uma síndrome pouco diagnosti- 
cada pela medicina e menos ainda conhecida por psicólogos. O melhor esclarecimento do diagnóstico, aliado ao aumento do conhecimento da etiologia, poderá resultar em aperfeiçoamento das intervenções.

Por fim, esta pesquisa teve como objetivo a realização de um exercício de análise funcional em uma portadora da SII. Algumas relações funcionais entre as atividades da vida cotidiana e os sintomas foram evidenciadas. Tais relações podem ser explicadas por meio do paradigma operante para a participante Sofia, mas outros casos poderiam ser explicados por outros modelos. Acredita-se que um modelo não é suficiente para explicar todos os casos, principalmente na SII, que pode ter diversas etiologias.

\section{REFERÊNCIAS}

Bennett, E. J., Tennant, C. C., Piesse, C., Badcock, C. A., \& Kellow, J. E. (1998). Level of chronic life stress predicts clinical outcome in irritable bowel syndrome. Gut, 43, 256-261.

Bohm, C. H. (2009). Síndrome do intestino irritável: Um exercício em análise funcional do comportamento. Dissertação de mestrado não-publicada, Universidade de Brasília.

Bohm, C. H., \& Gimenes, L. S. (2008). Automonitoramento como técnica terapêutica e de avaliação comportamental. Revista Psicolog, 1, 88-100.

Boyce, P., Gilchrist, J., Talley, N. J., \& Rose, D. (2000). Cognitive-behaviour therapy as a treatment for irritable bowel syndrome: A pilot study. Australian and New Zeland Journal of Psychiatry, 34, 300-309.

Chang, L., \& Heitkemper, M. M. (2002). Gender diferences in irritable bowel syndrome. Gastroentereology, 123, 1686-1701.

Corney, R. H., Ruth, S., N., Robert, N., \& Anthony, C. (1991). Behavioural psychotherapy in the treatment of irritable bowel syndrome. Journal of Psychosomatic Research, 35, 461-469.

Drossman, D. A. (2006). The functional gastrointestinal disorders and the Rome III process. Gastroentereology, 130(5), 13771390.

Fernandez, C., Perez, M., Amigo, I., \& Linares, A. (1998). Stress and contingency management in the treatment of irritable bowel syndrome. Stress Medicine, 14, 31-42.

Frederiksen, L. W. (1975). Treatment of ruminative thinking by self-monitoring. Journal of Behavior Therapy and Experimental Psychiatry, 6, 258-259.

Gimenes, L. S. (1997). Comportamento adjuntivo: Um possível modelo para a análise e intervenção em problemas de saúde. Em R. A. Banaco (Org.), Sobre comportamento e cognição: Vol. 1. Aspectos teóricos, metodológicos e de formação em análise do comportamento e terapia cognitivista (pp. 395-403). São Paulo: ARBytes.

Gimenes, L. S. (2003). Contingência conseqüencial: Expansão dos termos para compreensão do comportamento [Resumo]. Em Associação Brasileira de Psicoterapia e Medicina Comportamental (Org.), Anais do XII Encontro Brasileiro de Psicoterapia e Medicina Comportamental. Londrina: ABPMC.

Gimenes, L. S., Andronis, P. T., \& Goldiamond, I. (1987). Estudo de algumas variáveis de procedimento na defecação induzida por esquemas de reforçamento. Psicologia: Teoria e Pesquisa, 3, 104-116.

Gimenes, L. S., Andronis, P. T., \& Goldiamond, I. (1988). Defecação induzida por esquema múltiplo de reforçamento. Ciência e Cultura, 40 (11), 1121-1123.

Gimenes, L. S., Andronis, P. T., \& Layng, T. V. (2005). O questionário construcional de Goldiamond: Uma análise não-linear de contingências. Em: H. J. Guilhardi \& N. C. Aguirre (Orgs.), Sobre comportamento e cognição: Vol. 15. Expondo a variabilidade (pp. 308-322). Santo André: ESETec.

Gimenes, L. S., Brandão, A. M., \& Benvenuti, M. F. (2005). Comportamento adjuntivo: Da pesquisa à aplicação. Em J. Abreu-Rodrigues \& M. R. Ribeiro (Orgs.), Análise do Comportamento: Pesquisa, teoria e aplicação. (pp. 99-112). Porto Alegre: ArtMed.

Goldiamond, I. (1974). Toward a constructional approach to social problems. Behaviorism, 2, 1-84.

Haynes, S. N., \& O'Brien, W. H. (1990). Functional analysis in behavior therapy. Clinical Psychology Review, 10, 649-668.

Hildebrandt, T., \& Latner, J. (2006). Effect of self-monitoring on binge eating: Treatment response or 'binge drift'? European Eating Disorders Review, 14(1), 17-22.

Johnson, W. G., Schlundt, D. G., Barclay, D. R., Carr-Nangle, R. E., \& Engler, L. B. (1995). A naturalistic functional analysis of binge eating. Behavior Therapy, 26(1), 101-118.

Korotitsch, W. J., \& Nelson-Gray, R. O. (1999). An overview of self-monitoring research in assessment and treatment. Psychological Assessment, 11(4), 415-425.

Lackner, J. M., Mesmer, C., Morley, S., Dowzer, C., \& Hamilton, S. (2004). Psychological treatments for irritable bowel syndrome: A systematic review and meta-analysis. Journal of Consulting and Clinical Psychology, 72(6), 1100-1113.

Latimer, P. R. (1988). Distúrbios gastrintestinais funcionais: Um enfoque de medicina comportamental. São Paulo: Andrei Editora.

Leibbrand, R., \& Hiller, W. (2003). Cognitive behavior therapy for functional gatrointestinal disorders: Is group treatment effective? Acta Neuropsychiatrica, 15, 242-248.

Lynch, P. N., \& Zamble, E. (1989). A controlled behavioral treatment study of irritabe bowel syndrome. Behavior Therapy, 18, 70-83.

Mayer, E. A., Nalibof, B. D., Chang, L., \& Coutinho, S. V. (2001) Stress and the gastrointestinal tract V. Stress and irritable bowel syndrome. American Journal of Physiology-Gastrointestinal and Liver Physiology, 280, 519-524.

Mizputen, S. J., Mendes, A. Magalhães, A. F. N., André, E. A., Alves, J. G., Silveira Júnior, L. S., Brito, M. V. H., \& Correia, R. A. (2006). História dos critérios diagnósticos da síndrome do intestino irritável. Em F. A. Quilici, C. F. Francesconi, M. C. F. Passos, M. T. Haddad \& S. J. Mizputen (Orgs.), Síndrome do intestino irritável: Uma visão integrada (pp. 51-90). São Paulo: Segmento Farma.

Neff, D. F., \& Blanchard, E. B. (1987). A multi-component treatment for irritable bowel syndrome. Behavior Therapy, 18, 7083.

Nelson, R. O., \& Hayes, S. C. (1981). Theoretical explanations for reactivity in self-monitoring. Behavior Modification, 5, 3-14.

Paclawskyj, T. R., Matson, J. L., Rush, K. S., Smalls, Y., \& Vollmer, T. R. (2000). Questions About Behavioral Function (QABF): A 
behavioral checklist for functional assessment of aberrant behavior. Research in Developmental Disabilities, 21, 223-229.

Passos, M. C. F., Filho, A. L., Pontes, E. L., Amarante, H. M. B. S., Eisig, J. N., \& Almeida, J. R. (2006). Introdução. Em F. A. Quilici, C. F. Francesconi, M. C. F. Passos, M. T. Haddad \& S. J. Mizputen (Orgs.), Síndrome do intestino irritável: Uma visão integrada (pp. 9-22). São Paulo: Segmento Farma.

Quilici, F. A., \& André, A. B. (2000). Introdução. Em F. A. Quilici \& A. B. André (Orgs.), Síndrome do intestino irritável: Um consenso nacional (pp. 9-11). São Paulo: Lemos Editorial.

Quilici, F. A., Francesconi, C. F. Haddad, M. T. Passos, M. C. F., \& Mizputen, S. J. (2006). Tratamento. Em F. A. Quilici, C. F. Francesconi, M. C. F. Passos, M. T. Haddad \& S. J. Mizputen (Orgs.), Síndrome do intestino irritável: Uma visão integrada (pp. 91-104). São Paulo: Segmento Farma.

Saito, Y. A., Schoelnfeld, P., \& Locke, G. R. (2002). The epidemiology of irritable bowel syndrome in North America: A systematic review. American Journal of Gastroentereology, 97(8), 1910-1915.

Samson, D. M., \& McDonnell, A. A. (1990). Functional analysis and challenging behaviours. Behavioural Psychotherapy, 18, 259-271.

Sidman, M. (2003). Coerção e suas implicações (M. A. P. A. Andery \& T. M. A. P. Sério, Trad.). São Paulo: Livro Pleno. (Original publicado em 1989)
Spiegel, B. M. R., Gralnek, I. M., Bolus, R., Chang, L., Dulai, G. S., Mayer, E. A., \& Naliboff, B. (2004). Clinical determinants of health-related quality of life in patients with irritable bowel disease. Archives of Internal Medicine, 164, 1773-1780.

Spollett, G. R. (1989). Irritable bowel syndrome: Diagnosis and treatment. Nurse Practitioner, 14(8), 32-44.

Suls, J., Wan, C. K., \& Blanchard, E. B. (1994). A multilevel dataanalytic approach for evaluation of relationships between daily life stressors and symptomatology: Patients with irritable bowel syndrome. Health Psychology, 13(2), 103-113.

Whitehead, W. E., \& Bosmajian, L. S. (1982). Behavioral medicine approaches to gastrointestinal disorders. Journal of Consulting and Clinical Psychology, 50(6), 972-983.

Whitehead, W. E., Renault, P. F., \& Goldiamond, I. (1975). Modification of human gastric acid secretion with operantconditioning procedures. Journal of Applied Behavior Analysis, $8(2), 147-156$.

Virués-Ortega, J., \& Haynes, S. N. (2005). Functional analysis in behavior therapy: Behavioral foundations and clinical application. International Journal of Clinical and Health Psychology, 5(3), 567-587.

Nota:

1 Pesquisa realizada com o apoio da Comissão de Aperfeiçoamento de Pessoal de Nível Superior (CAPES) e do Fundo de Pesquisa do Decanato de Pesquisa e Pós-graduação da Universidade de Brasília.

\section{Sobre os autores:}

Carlos Henrique Bohm: Graduado em Psicologia pela Universidade Estadual de Maringá (2006) e Mestre em Ciências do Comportamento pela Universidade de Brasília (2009). Atualmente cursa doutorado em Ciências do Comportamento na Universidade de Brasília.

Lincoln da Silva Gimenes: Doutor em Biopsicologia pela University of Chicago (1986). Pós-doutorado na Oregon Health \& Science University na área de Toxicologia Comportamental (1998). Professor titular da Universidade de Brasília.

Endereço para correspondência: Carlos Henrique Bohm. SCLRN 714, Bl. F, Ent. 11, Ap. 102 - 70760-556 Brasília/DF. Endereço eletrônico: bohm.carlos@gmail.com. 